\title{
Using nano zero valent iron supported on diatomite to remove acid blue dye: synthesis, characterization and toxicology test
}

\author{
Erick Justo-Cabrera ${ }^{1}$, Ernesto Flores-Rojas ${ }^{2}$, Denhi Schnabel ${ }^{1}$, Héctor Poggi-Varaldo ${ }^{2}$, \\ Omar Solorza-Feria ${ }^{2}$, Luz Breton-Deval*3
}

1- Instituto de Biotecnología, Universidad Nacional Autónoma de México, Avenida Universidad 2001, Colonia Chamilpa, Cuernavaca, Morelos 62210, México

2- Centro de Investigación y de Estudios Avanzados del Instituto Politécnico Nacional. Av. Instituto Politécnico Nacional 2508, Col. San Pedro Zacatenco, Delegación Gustavo A. Madero, México D.F

3- Catedras-Conacyt, Instituto de Biotecnología, Universidad Nacional Autónoma de México, Avenida Universidad 2001, Colonia Chamilpa, Cuernavaca, Morelos 62210, México

\section{*Corresponding author:}

Dr. Luz Breton-Deval

E-mail: lzbreton@ibt.unam.mx

\section{CATEDRAS-CONACYT}

Instituto de Biotecnología, Universidad Nacional

Autónoma de México, Avenida Universidad 2001, Colonia Chamilpa, Cuernavaca, Morelos 62210, México. 


\section{Using nano zero valent iron supported on diatomite to remove acid blue dye: synthesis, characterization and toxicology test}

\section{Abstract}

The aim of this work was to synthesize and characterize nanoscale zero-valent iron (NZVI) supported on diatomaceous earth (DE) at two different molar concentration $3 \mathrm{M}$ and 4M (nZVI-DE-1 nZVI-DE-2), to test the decolorization treatment of acid blue dye (AB) and perform a toxicological test using zebrafish. The synthesis of the nanoparticles was obtained using the chemical reduction method and the material was characterized by X-ray diffraction, Scanning Electron Microscopy (SEM), Energy-Dispersive X-Ray (EDX), and transmission electron microscopy and Specific Surface Area (BET). The results showed spherical forms in clusters between 20 to $40 \mathrm{~nm}$ of zero valent iron supported on diatomaceous earth. The removal of $1 \mathrm{~g} / \mathrm{l}$ of $\mathrm{AB}$ from water treated with NZVI-DE-1 and NZVI-DE-2 reached the decolorization of $90 \%$ and $98 \%$ of all dye. While controls like NZVI and DE-1 and DE-2 achieved the removal of 40, 37 and $24 \%$ of the dye. Toxicological analysis using zebrafish showed that $A B$ causes a severe defect in development and embryos die after exposure. However, the water samples treated with NZVI-DE-1 and NZVI-DE-2 are not harmful for the zebrafish embryos during the first 24 hours. We conclude that the use of NZVI-DE-1 and NZVI-DE-2 is a promising treatment for dye pollution.

\section{Keywords: nanoparticles, water treatment, dyes, zero-valent iron, acid blue}




\section{Introduction}

The concentration of dyes in industrial effluents can reach $500 \mathrm{mg} / \mathrm{L}$ and pollute local freshwater, reducing the efficiency of sunlight and thereby impeding the process of photosynthesis. (Jung et al., 2016). As a result, the water temperature of the stream decreased, and photoautotrophs organism like algae, euglena, and cyanobacteria could no longer survive (Bide, 2007). The death of these organisms is an ecological loss because they play an essential role in the cycle of nutrients and are capable of absorbing organic matter present in the stream. They can also remove carbon dioxide from the atmosphere, are an indispensable source of food and oxygen for several organisms (Callieri, 2014). Pollution due to synthetic dyes also affects the health of aquatic and terrestrial organisms (Samchetshabam et al., 2017).

Acid blue (AB) was selected for this study because it is used widely by the industrial sector, including for cosmetics, food coloring or for dyeing different fibers, and is commonly mixed with sulfuric acid to make it more soluble before industrial application thereby increasing the toxicity (Ammar et al., 2006). AB is not only toxic for aquatic life but can also cause skin irritation, cornea damage, and promote the development of tumors and cancer (Khelifi et al., 2008).

Several kinds of research have been carried out with the aim of removing dyes from water using aerobic or anaerobic degradation, filtration, adsorption, membrane filtration and other methods (Pacheco-Álvarez et al., 2019; Saleh, 2019; Wang et al., 2018). However, all of these processes have disadvantages such as the extended time required for the treatment, high operational costs, and efficiency (Ziarani et al., 2018).

Recently, nanomaterials have been used to remove several pollutants including dyes such as crystal violet (Răducan et al., 2019), methyl orange (Fan et al., 2009), acid red 88 and Black 5 (Sharma and Shirkot, 2019). 
Materials made of nanoparticles have a relatively high surface area to mass ratio rendering them more reactive compared to the conventional materials used for water treatment (Quinn et al., 2005). However, this technology has to overcome some challenges in order to be applied successfully; namely that during synthesis the nanoparticles tend to aggregate easily thereby losing surface area (Raychoudhury et al., 2012; Zhang et al., 2013) and becoming toxic for aquatic life (Krysanov et al., 2010). To avoid these problems, we supported the nanoparticles on diatomite, a well-known filter material in the industry, and completed a toxicological test using zebrafish.

Zebrafish (Danio rerio) have been established as an ideal model for toxicological studies to test the effects of contaminants such as alkaloids, glycosides, metals, alcohols, carboxylic acids, among others (Chen et al., 2011; Qiang and Cheng, 2019). Zebrafish have a number of characteristics that make them a good model for testing toxicity. Firstly, female zebrafish are able to produce hundreds of eggs and embryos are transparent which allows for close observation of development under microscope. Secondly, the rapid growth of zebrafish compared to other vertebrates makes it an ideal model for highthroughput analysis (Ali et al., 2011).

Therefore, the objective of this research was to characterize and synthesize nanoscale zero-valent iron supported on diatomaceous earth and to test if treated water had a negative effect on the viability of zebrafish embryos.

\section{Materials and Methods}

\section{Preparation of diatomite earth and synthesis of nanomaterial}

The diatomite earth (DE) was washed with $1 \mathrm{M} \mathrm{HCl}$ for $8 \mathrm{~h}$ under agitation (150 rpm). After the acid-washed, the DE was rinsed with water, before using as NZVI support. 
121 Two types of NZVI-DE were synthesized. The first identified as NZVI-DE-1 was prepared from $0.3 \mathrm{M} \mathrm{FeCl}_{2} .4 \mathrm{H}_{2} \mathrm{O}$ at a $40 / 60$ proportion of $\mathrm{FeCl}_{2} .4 \mathrm{H}_{2} \mathrm{O} / \mathrm{DE}$, while the second type identified as nZVI-DE-2 was made at a 50/50 proportion of $\mathrm{FeCl}_{2} \cdot 4 \mathrm{H}_{2} \mathrm{O}$ / DE. NZVI and NZVI-DE-1 and 2 were obtained using the chemical reduction method of $\mathrm{FeCl}_{2} .4 \mathrm{H}_{2} \mathrm{O}$ in aqueous solution using $\mathrm{NaBH}_{4}$ as a reducing agent, due to its simplicity and efficiency in securing NZVI (Fu et al., 2014). The $\mathrm{FeCl}_{2} .4 \mathrm{H}_{2} \mathrm{O}$ and DE were added to a previously ethanol deoxygenated by bubbling it with $\mathrm{N}_{2}$ gas for $30 \mathrm{~min}$. The iron salt was dissolved in $50 \mathrm{~mL}$ ethanol, and the solution was kept under $\mathrm{N}_{2}$ bubbling and stirring for $30 \mathrm{~min} .400 \mathrm{rpm}$ at $25^{\circ} \mathrm{C}$. Later, $1.5 \mathrm{M} \mathrm{NaBH}_{4}$ solution was slowly added into the $\mathrm{FeCl}_{2} / \mathrm{DE}$ solution. After the reaction, the solution was kept stirring at $700 \mathrm{rpm}$ for 60 min. In the end, both types of NZVI-DE were washed 10 times with ethanol and dried in an oven, bubbling with argon at $50{ }^{\circ} \mathrm{C}$. The synthesis of the NZVI without DE support followed the same technique.

\section{Characterization of iron nanoparticles}

\section{X-Ray Diffraction (XRD)}

The XRD analysis was carried out using a Bruker D8 Advance Eco diffractometer coupled with a copper source without a monochromator; the samples were placed in a sample holder with a $2 \theta$ range of $5^{\circ}-130^{\circ}$ at a size and time of $0.02^{\circ}$ and 0.2 seconds. The analysis of the crystalline phases was carried out using Match software.

\section{Scanning Electron Microscopy (SEM) with Energy-Dispersive X-Ray (EDX)}

A scanning electron microscope (SEM) HRSEM-AURIGA Zeiss integrated with an Xray scattered energy (EDX) analyzer at a voltage of 1-10 keV at high vacuum was used. 
random areas using the EDX analyzer. The samples were placed on graphite tape supported on metal discs.

\section{Transmission Electron Microscopy (TEM)}

A transmission electron microscope (TEM) JEM-ARM200F operated at a high vacuum was used to describe the morphology, size, and distribution of the particles. The samples were prepared by dispersing a small amount of powder in absolute ethanol with the aid of an ultrasonic bath. The dispersion $(10 \mu 1)$ was applied on a copper mesh for TEM of 300 mesh with Lacey / Carbon film and allowed to evaporate in a desiccator.

\section{Specific Surface Area (BET)}

The specific surface area was measured using the Brunauer-Emmett-Teller (BET) method of $\mathrm{N}_{2}$ adsorption, using a Micromeritics Gemini 2360 surface area analyzer. Before analysis, the samples were degassed under vacuum at $70{ }^{\circ} \mathrm{C}$ for three hours.

\section{Batch degradation experiments}

The experimental design consisted of a factorial analysis to evaluate two different relationships between the quantity of NZVI and DE (NZVI-DE-1 $=40 / 60$, and NZVIDE-2 $=50 / 50)$ but hold the same quantity of iron $(62 \mathrm{mg})$ in every treatment. The experiment was carried out using serum bottles with $50 \mathrm{~mL}$ of water contaminated with dye (1000 mg/L) loaded with $658 \mathrm{mg}$ of Nano NZVI-DE. The controls were (i) DE-1 and DE-2 to evaluate dye degradation to adsorption process (ii) NZVI to assess the effect of free nanoparticles without a support and (iii) AD to calculate the abiotic degradation. The 
performed in triplicate. The removal of $A B$ was measured with the UV spectra (BioSpectrometer, Eppendorf) at $526 \lambda \mathrm{nm}$.

\section{Fish maintenance and strains}

$174 \mathrm{AB}$ line and wild-type zebrafish (Danio rerio) embryos were obtained from natural 175 crosses and raised at $28{ }^{\circ} \mathrm{C}$ based on standard procedures (Westerfield, 2000). Eggs were obtained by random pairwise mating of zebrafish. The eggs were harvested the following morning and transferred into plastic Petri dishes (60 eggs per dish) containing $10 \mathrm{ml}$ fresh embryo water. Further, unfertilized, unhealthy, and dead embryos were identified under a dissecting microscope and removed. Morphological criteria determined embryonic stages, according to Kimmel and collaborators (Kimmel et al., 1995). Zebrafish were handled in compliance with local animal welfare regulations, and all protocols were approved by the ethics committee (Instituto de Biotecnología, UNAM).

\section{Toxicological studies}

At 3.5 hours post-fertilization (hpf), embryos were again screened, and any additional dead and unhealthy embryos were removed. Embryos at the sphere stage were selected and transferred into a 48 well plate; ten in each well. Later the embryo water was absorbed, and $300 \mathrm{ul}$ of the treated water samples (AB dye, NZVI-DE-1, NZVI-DE-2, DE-1, DE-2) were loaded into each well; experiments were performed in triplicate. The control was a set of embryos grown with embryonic water in the same plate as well as in a petri dish independently. The embryonic plates were cultivated in a moist chamber at $28{ }^{\circ} \mathrm{C}$ from 6 to $24 \mathrm{hpf}$, and the viability of the embryos was observed under a dissecting microscope. Embryos at $24 \mathrm{hpf}$ were anesthetized with tricaine, immobilized with methylcellulose on agar plates and visualized with a stereomicroscope (Leica MZ 12.5), 
Statistical analysis was performed with Prism (GraphPad) Student's t-test. Error bars in column graphs represent the standard deviation of the mean (s.d.).

\section{Results and Discussion}

\section{Synthesis and Characterization of iron nanoparticles}

The SEM/EDX analysis of DE showed high levels of oxygen at around $52 \%$ given that most of the materials that form DE are oxides, such as silicon oxide or aluminum oxide (Table 1).

Table 1 Element composition of the diverse treatments DE, NZVI, NZVI-DE1 and NZVI-DE2

\begin{tabular}{ccccc}
\hline Element & DE & NZVI & NZVI-DE1 & NZVI-DE2 \\
\hline $\mathrm{Si}$ & $36.7 \pm 1.1$ & ND & $23.9 \pm 2.9$ & $23.3 \pm 0.6$ \\
$\mathrm{Al}$ & $8.6 \pm 1.5$ & ND & $8.4 \pm 0.2$ & $7.0 \pm 0.3$ \\
$\mathrm{O}$ & $52.1 \pm 0.1$ & 19.27 & $43.2 \pm 2.2$ & $42.1 \pm 0.3$ \\
$\mathrm{~S}$ & $0.9 \pm 0.1$ & $\mathrm{ND}$ & $1.3 \pm 0.5$ & $1.2 \pm 0.3$ \\
$\mathrm{~K}$ & $0.5 \pm 0.1$ & $\mathrm{ND}$ & $0.7 \pm 0.3$ & $0.7 \pm 0.2$ \\
$\mathrm{Fe}$ & $\mathrm{ND}$ & 80.73 & $21.9 \pm 2.3$ & $24.9 \pm 0.5$ \\
\hline
\end{tabular}

The second most abundant element in DE is $\mathrm{Si}(37 \%)$ as was expected and elsewhere reported (Yuan et al., 2010). The Si content varies depending on the physicochemical conditions present during the formation of the DE bank (Guatame-Garcia, Buxton, 2018). These minor variations could affect the performance of the material because the higher the content of Si the more silanol groups available to react with polar organic compounds increasing the number of compounds adsorbed by DE (Al-Ghouti et al., 2003). Carbonate minerals can also affect the number of compounds that DE can absorb because the deposit of this material along the diatomite structure reduces its porosity (Guatame-Garcia, A. 

the DE with the abundance of cristobalite, tridymite, and quartz, but anyone with nanoparticles (Fig. 1).

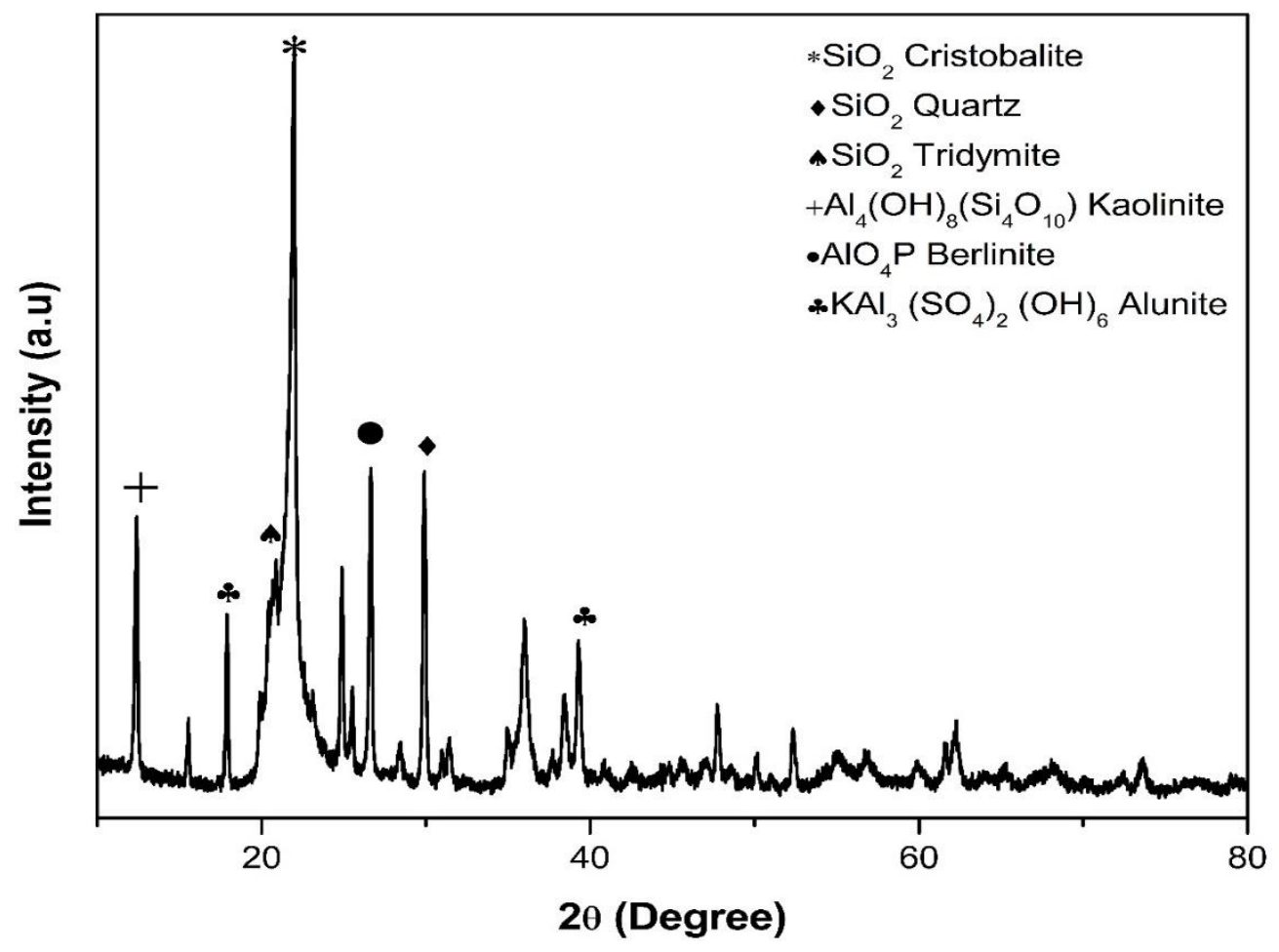

Fig. 1 XRD pattern of diatomaceous earth

The Brunauer-Emmett-Teller (BET) specific surface area was of $23 \mathrm{~m}^{2} / \mathrm{g}$ with a pore volume of $0.56 \mathrm{~cm}^{3} / \mathrm{g}$. As has been recorded by other DE characterization studies (Crane and Sapsford, 2018). The homogenous pore volume allows the molding of narrow size particles dispersed homogeneously on their internal surfaces (Machado et al., 2019). Regarding NZVI-DE-1 and NZVI-DE-2, the SEM/EDX analysis showed a decrease in the levels of $\mathrm{Si}$ and $\mathrm{O}$ compared with the $\mathrm{DE}$ analysis given that space is occupied by iron $22 \%$ and $25 \%$ for NZVI-DE-1 and NZVI-DE-2, respectively (Table 1). The XRD pattern of NZVI-DE showed diffraction peaks at the $2 \theta$ of $44.90^{\circ}$ confirming the presence of zerovalent iron in both treatments of NZVI-DE as well as on NZVI treatment (Fig. 2). 


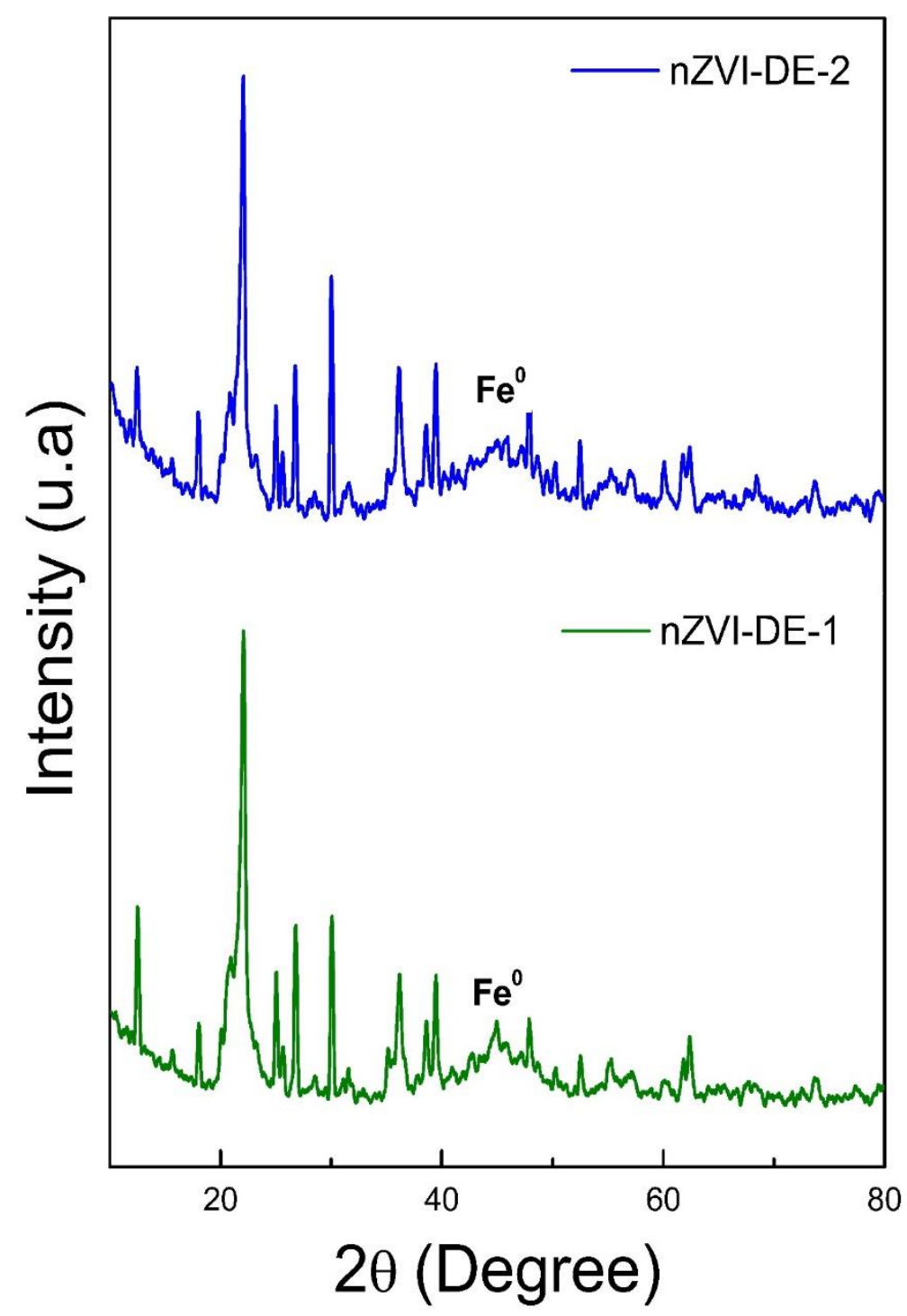

235 Fig. 2. XRD pattern of nZVI-DE-1 and nZVI-DE-2 treatments

236 Crane and Sapsford et al. have reported similar XRD patterns of iron and DE. However,

237 they indicate the presence of other metals such as Al, suggesting that perhaps the duration 238 of the acid wash $\sim 2 \mathrm{~h}$ was not enough (Crane and Sapsford, 2018). The TEM images 239 showed spherical forms in clusters between 20 to $40 \mathrm{~nm}$, with an average size of $35 \pm 8$ nm (Fig. 3).

\section{Batch degradation experiments}

243 The reaction proceeded in the first 5 min with a drastic change of color, and later, the 244 difference decelerated and stopped at minute 7. Fig. 4 shows the UV-vis spectra taken 10 

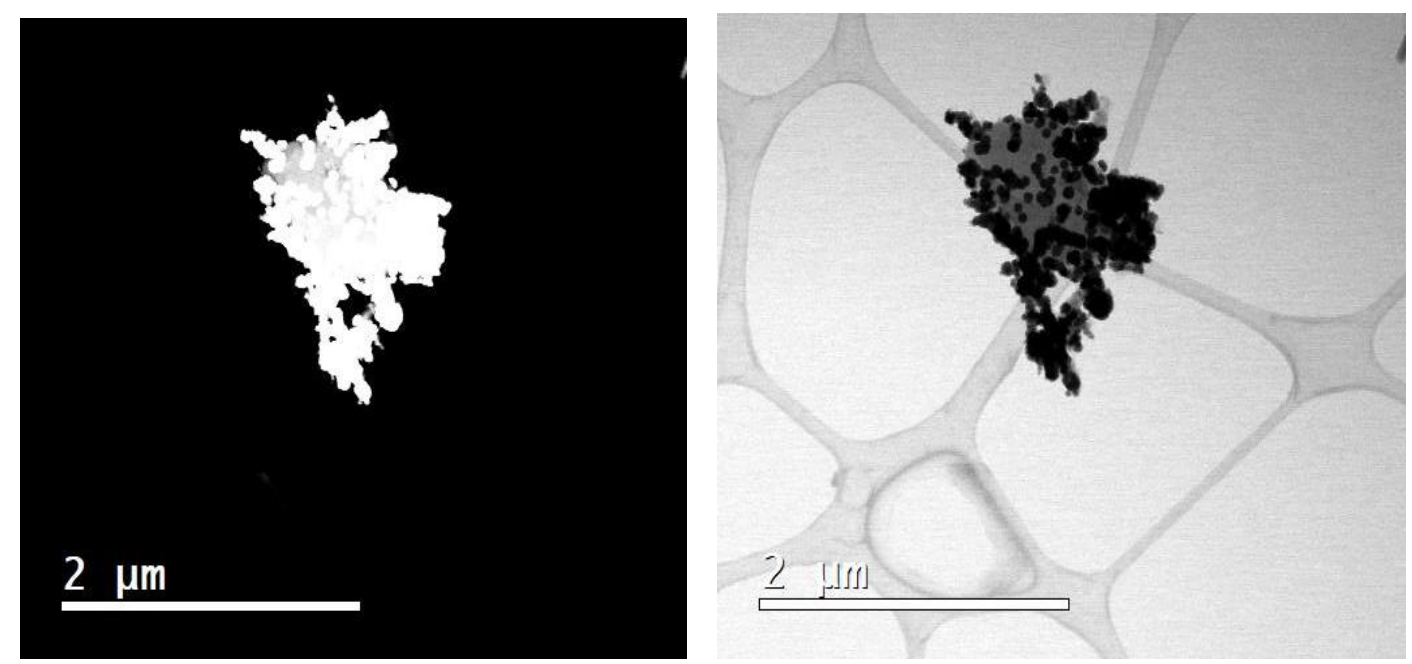

252

Fig. 3. Dark field (A) and bright field (B) micrographs of the Fe nanoparticles decorating

min. after, when the reaction was stable. The black line shows the spectra of the water

with $1 \mathrm{~g} / \mathrm{L}$ of $\mathrm{AB}$, the red line indicates the NZVI-DE-1 treatment which removed $90 \%$

of the pigment, and the blue line shows that the NZVI-DE-2 treatment achieved $98 \%$

removal of $\mathrm{AB}$. The NZVI control removed just $40 \%$ of $\mathrm{AB}$, and DE- 1 and DE-2 controls

removed $37 \%$ and $24 \%$ respectively. The contrast between the catalytic power of supported and unsupported nanomaterials is significant NZVI-DE-1 and NZVI-DE-2 removed 50 and $58 \%$ more dye than unsupported nanoparticles.

In NZVI-DE-1 and NZVI-DE-2, the NZVI particles were responsible for the removal of the dye through reduction and adsorption processes (Deng et al., 2018; Fan et al., 2009), while the DE played a protective role for the NZVI catalyst (reducing agglomeration) and contributed to the adsorption of $\mathrm{AB}$. The amount of $\mathrm{AB}$ removed and adsorbed could be calculated based on removal results of NZVI and DE controls, and evidently, around 40 $-50 \%$ of the removal was adsorbed by the DE and the rest was the reduction performed by the nanoparticles. Another factor that could contrast the removal of both treatments 


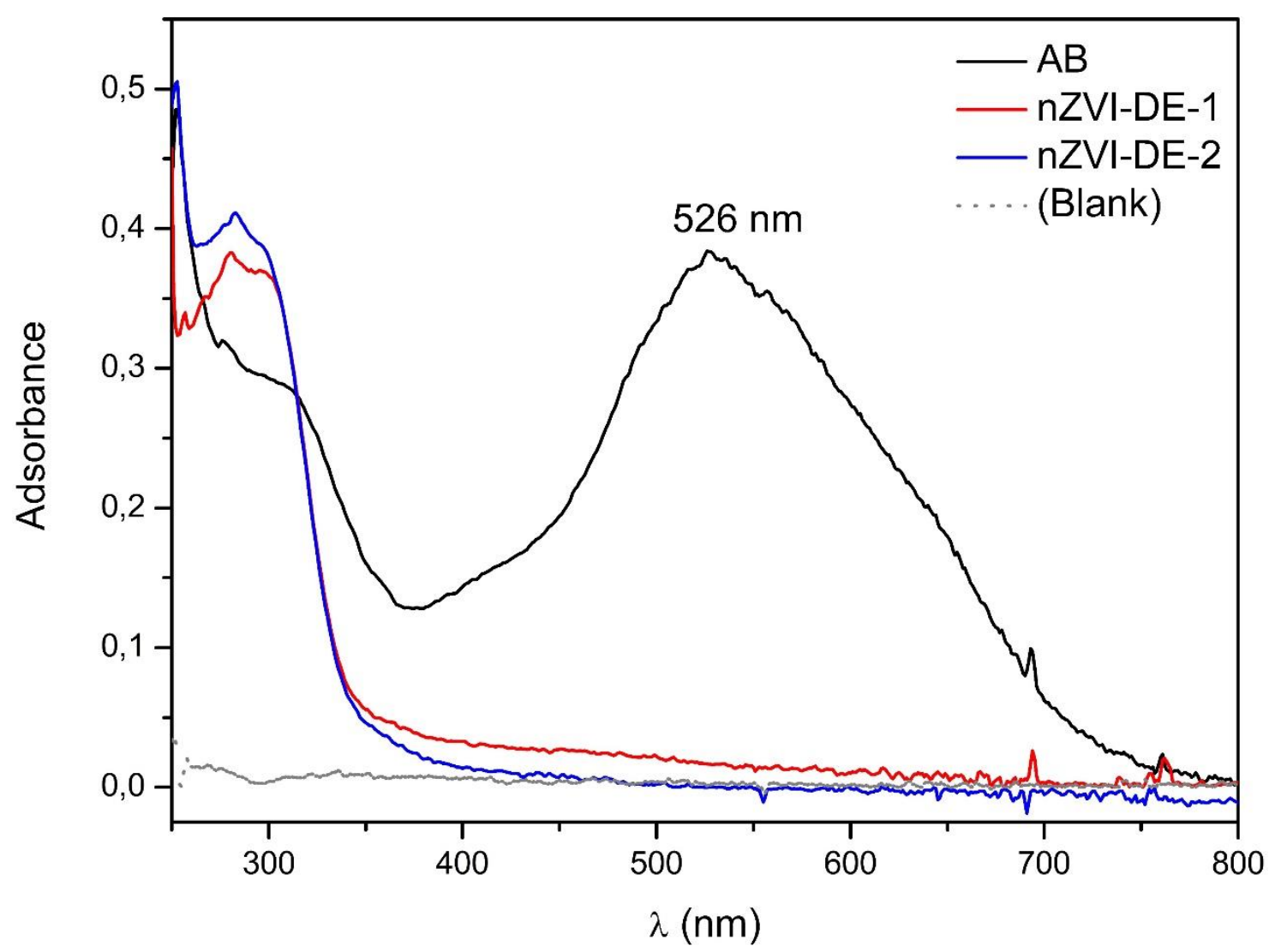

Fig. 4 Absorbance signal of the dye and the dye removed by the NZVI-DE-1 and 2

could be the amount of DE. NZVI-DE-1 had less DE where nanoparticles were supported; and this treatment also had less iron than NZVI-DE-2. The removal performed by the nanoparticles in contact with the contaminant occurs because the nanoparticles play a role as electron mediator; also, $\mathrm{H}$ atoms are generated and this causes the break of the double bond $(-\mathrm{C}=\mathrm{C}-$ ) eliminating the chromophore group of the dye (Chen et al., 2011). Destroying one of the critical components of the dye and, as a result, the absorption peak at a wavelength of $526 \mathrm{~nm}$ was reduced (Fig. 4). At the same time, $\mathrm{Fe}^{\circ}$ reacted and formed oxides such as $\mathrm{FeO}_{2}, \mathrm{FeO} 3$, and $\mathrm{FeOH}$ which have a high capacity to absorb some molecules of the pollutant (Wu et al., 2015). However, this capacity to absorb could be affected by internal changes in $\mathrm{pH}$ (Shah et al., 2015). The $\mathrm{pH}$ of the dye solution plays an essential role in the entire adsorption process and the adsorption capacity, influencing the loads of the NZVI-DE and DE. This changes the degree of ionization and dissociation 
of the functional groups in the active sites of the adsorbent materials (Basheer, 2018). At the beginning, the $\mathrm{pH}$ of the treatments was 7.4 , however at the end of the removal experiments, NZVI-DE-1 had 8.9; and NZVI-DE-2 had 8.7; while NZVI had a more neutral $\mathrm{pH}$ of 7.7; and DE-1 and 2 had an acid $\mathrm{pH}$ of 4 and 4.6., respectively. The treatment with nanoparticles showed $\mathrm{pH}$ values around 8 because the dye removal is a dynamic process where $\mathrm{Fe}^{\circ}$ nanoparticles begin to be transformed into oxides as $\mathrm{Fe}^{2+}$, $\mathrm{Fe}^{3+}, \mathrm{Fe}(\mathrm{OH})_{3}$ and $\mathrm{Fe}(\mathrm{OH})_{2}$, which reduces the quantity of $\mathrm{H}+$ and raises the $\mathrm{pH}$ of the liquid (Garg et al., 2018). Whereas in the treatment with only DE, the $\mathrm{pH}$ of the solutions finishes acidic due to the protonation of surface silanol groups where protons are forming conjugate acids that lower the pH (Lowe et al., 2015; Nosrati et al., 2017).

\section{Toxicological analysis of treated water on zebrafish embryos}

297

Embryos were treated with AB, and the water treated with NZVI-DE-1, NZVI-DE-2, DEonly to AB died after treatment; meanwhile, embryos treated with NZVI-DE-1 and NVZIDE-2 developed alongside the embryos in control water (Fig. 5 A-C and G). Embryos grown in the presence of DE developed regularly (data not shown). The embryos treated with NZVI-DE-1 and NZVI-DE-2 were allowed to develop until 24 hpf; embryos developed normally, but a slight developmental delay was observed compared to the control (Fig.5 D-F). However, after 24 hours of culture particles associated with the chorion were visible in NZVI-DE-1 and NZVI-DE-2 samples, possibly due to an agglomeration of nanoparticles (Fig. $5 \mathrm{~B}$ and $\mathrm{C}$ asterisk), and perhaps this caused the developmental delay observed in these samples. At 48 and $72 \mathrm{hpf}$ (data not shown) there 
Control
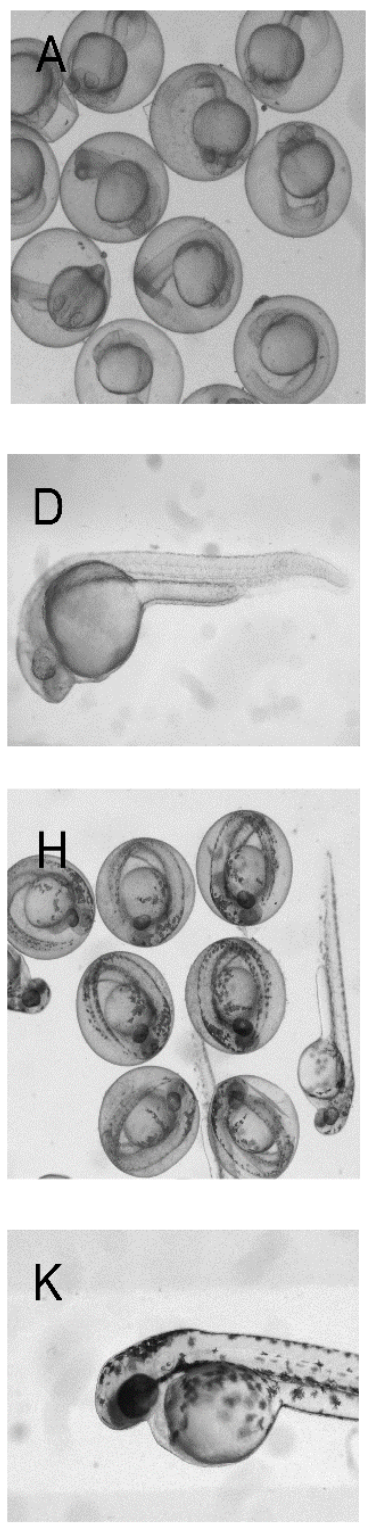

NZV-DE-1
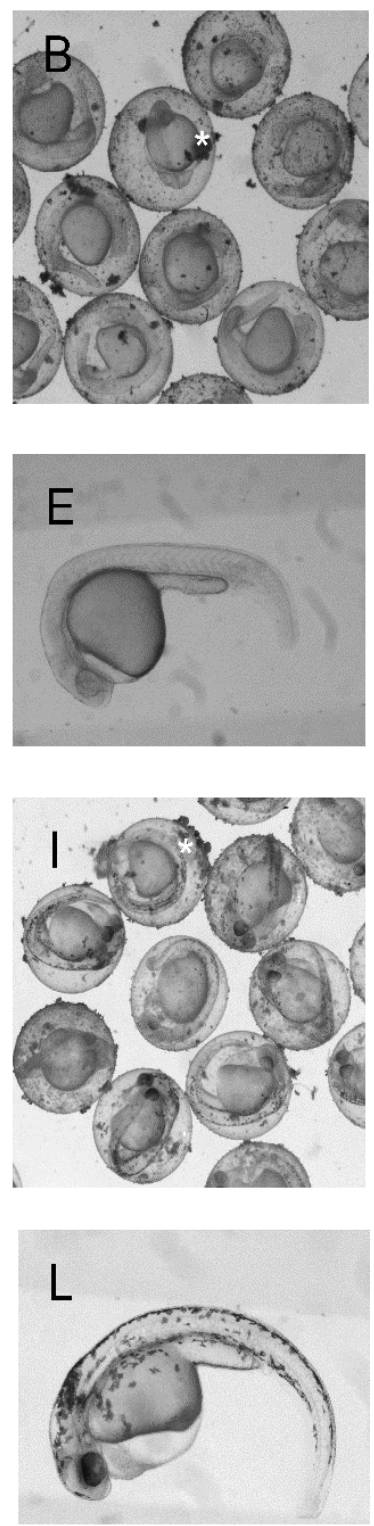

NZV-DE-2

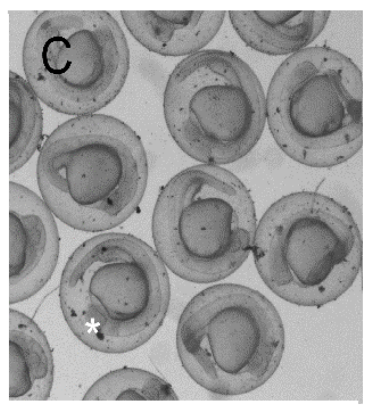

G
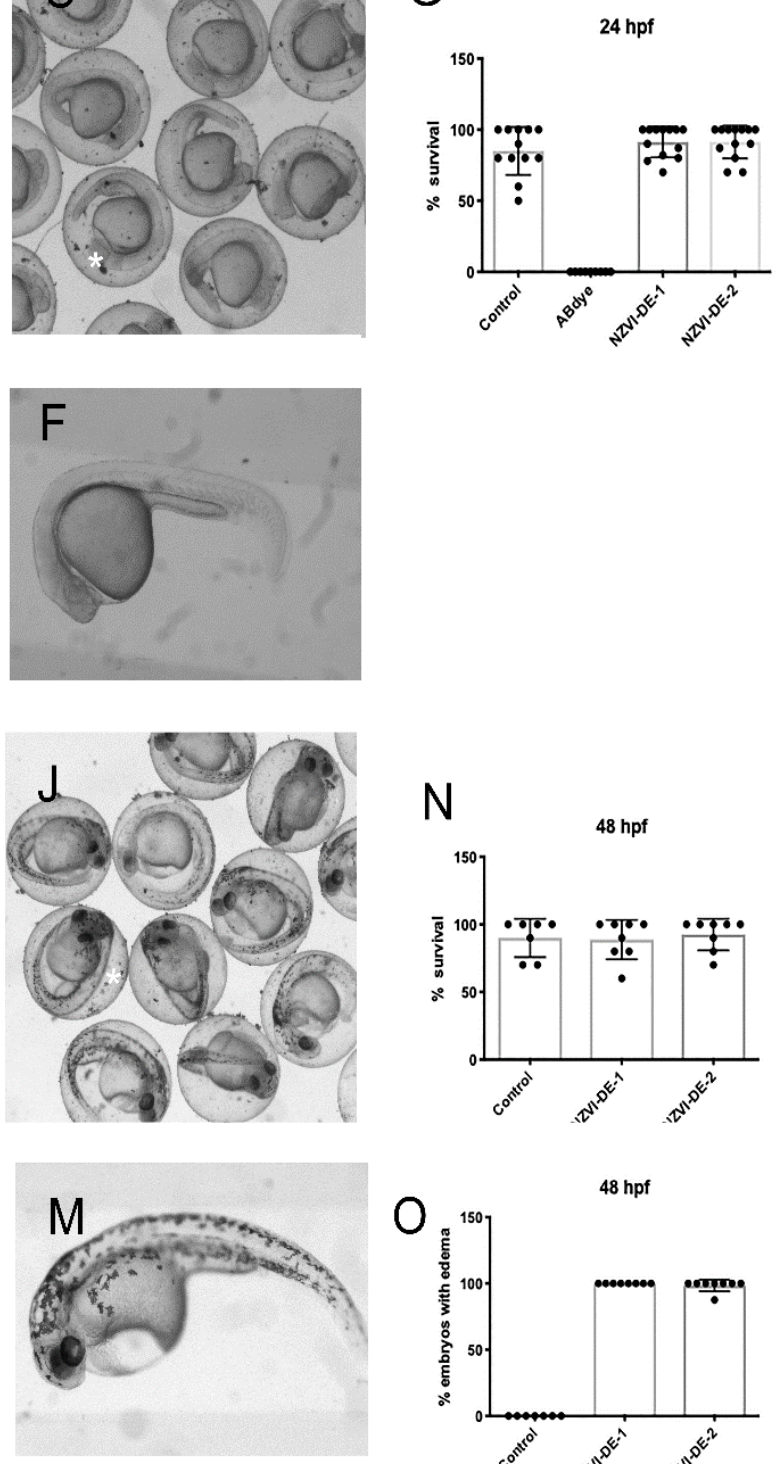

Fig. 5 Zebrafish embryonic development progress in the presence of NZV-DE-1 and
NZV-DE2. A. Control embryos at 24hpf. B and C. Embryos at 24hpf proceed with development when exposed to contaminated water is treated with NZV-DE. Agglomeration of nanoparticles can be observed in the chorion (asterisk in B and C). D-F. A slight delay in development is observed in NZV-DE-1 (E) and NZV-DE-2 (F) embryos compared to the control (D). $\mathbf{G}$ and $\mathbf{N}$ Viability is fully restored in NZV-DE treated water exposure compared to $\mathrm{AB}$ dye exposure. $\mathbf{H}$ Control embryos at $48 \mathrm{hpf}$. I and $\mathbf{J}$ Embryos developed until $48 \mathrm{hpf}$ as the control embryos; nevertheless, the embryos treated with NZV-DE (L and M) present cardiac edema $(\mathrm{O})$, smaller eyes curved body plan, less pigmentation and are smaller compared to the control $(\mathrm{K})$. 
embryos had cardiac edema, smaller eyes and curved and smaller bodies with less pigmentation (Fig. $5 \mathrm{H}-\mathrm{O}$ ). We also observed particles attached to the chorion (Fig $5 \mathrm{~B}$ and C; A and J); these could be the nanoparticles since we do not observe these aggregates in the control embryos. At $96 \mathrm{hpf}$ the embryos treated with the water from NZVI-DE-1 and NZVI-DE-2 died; meanwhile control embryos developed normally. The delay in development at $24 \mathrm{hpf}$; the morphological effect at 48 and $72 \mathrm{hpf}$ and the comprised viability of embryos at $96 \mathrm{hpf}$ could be due to the presence of the nanoparticles. Previous work done by Zhu X et al. (2012) showed that embryos exposed to iron nanoparticles caused effects in survival and morphological defects similar to what we observe, such as cardiac edema.

Nevertheless, the improvement of embryonic viability was highly significant compared to the embryos treated only with the $\mathrm{AB}$ dye (Fig.6). In order to determine the exact moment that the $\mathrm{AB}$ had an effect, dye cultures of 4 hours were carried out. Here we could observe that after 4 hours of treatment all of the embryos treated with $\mathrm{AB}$ dye were already dead; meanwhile control embryos and those treated with NZV1-DE-1 and NZV1DE-2 developed normally (Fig 6). Additionally, the embryos treated with AB dye were colored as was the chorion (Fig. 6D and H), while NZV1-DE-1 and NZV1-DE-2 treated embryos followed normal development without coloration (Fig. 6). Our results suggest that the treatment of NZV1-DE-1 or NZV1-DE-2 is sufficient to avoid the lethality observed in embryos exposed to $\mathrm{AB}$ dye given that development proceed normally at the first hours of treatment. Nevertheless, further stages of development, such as 48 and 72 hpf, were affected as a result of the nanoparticles present in the samples. The effects observed in the embryos after 24 hours could be mitigated by potential applications of NZVI-DE via filters where the treated water is in contact with the organism for a short period of time 
Control
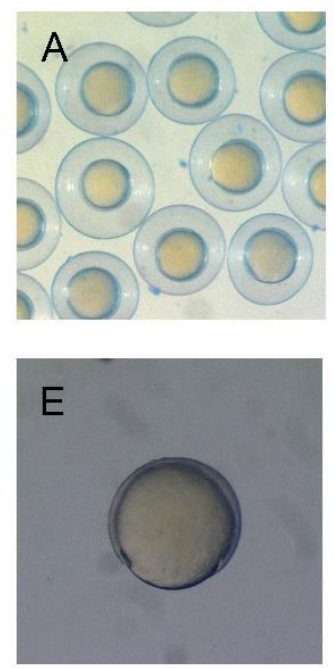

NZV-DE-1
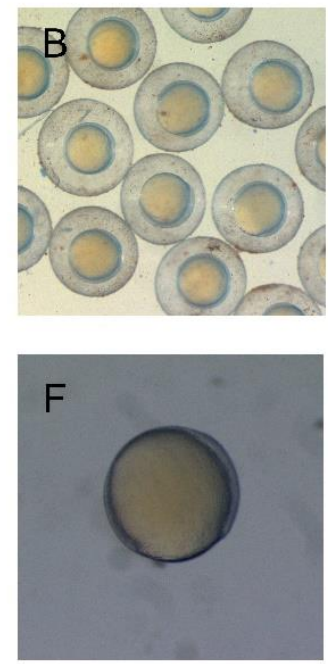

NZV-DE-2
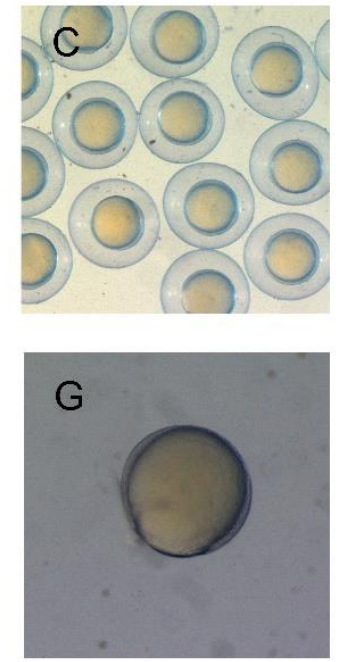

$A B$ dye
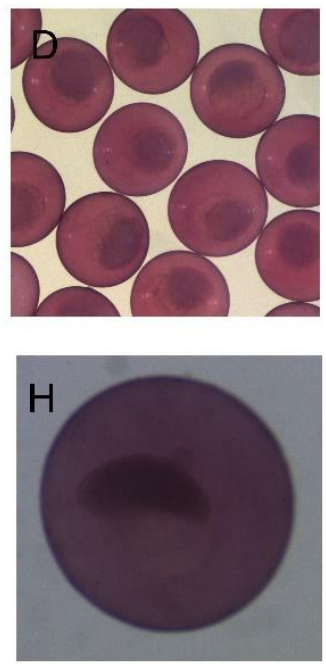

Fig. 6 Early zebrafish development is restored after NZV-DE treatment. A. Control embryos after 4 hours of treatment. B Embryos treated with NZV-DE-1 develop normally. C. Embryos treated with NZV-DE-2 develop as the control embryos. D Embryos treated with $\mathrm{AB}$ dye died after 4 hours of treatment. E-H Higher magnifications show that embryos treated with NZV-DE (F and G) develop as the control embryos (E); meanwhile embryos treated with $\mathrm{AB}$ dye absorb the dye and do not develop further and die.

\section{Conclusion}

There is increasing interest in the use of nanoparticles to remove pollutants, however the possible risk to aquatic life or through food chains are not sufficiently clear. In this experiment, the NZVI-DE-1 and NZVI-DE-2 successfully removed more than $90 \%$ of one of the most widely used pollutants, acid blue. The toxicology test of the treated water showed that wild-type zebrafish (Danio rerio) embryos developed completely normally during the first $24 \mathrm{~h}$. However, after $48 \mathrm{~h}$. all of the embryos had cardiac edema, smaller eyes, and smaller bodies with less pigmentation compared to the control sample. Consequently, we suggest that nanoparticles technologies use supported materials and that nanoparticle technologies used to remove pollutants have limited contact with streams in order to avoid adverse effects for aquatic life. 


\section{Acknowledgements}

The authors thank ZEOLITECH for the donation of diatomaceous earth, Dr. Daniel Bahena Uribe and Dr. Jorge Roque De La Puente of LANE, CINVESTAV, for their excellent technical help and advice on the characterization of nanoparticles. Also, LB-D thanks the Consejo Nacional de Ciencia y Tecnología (CONACYT) and their program CATEDRAS for their support of the Project 285.

\section{Reference}

Al-Ghouti, M.A., Khraisheh, M.A.M., Allen, S.J., Ahmad, M.N., 2003. The removal of dyes from textile wastewater : a study of the physical characteristics and adsorption mechanisms of diatomaceous earth. Journal of Environmental Management 69, 229-238. doi:10.1016/j.jenvman.2003.09.005

Ali, S., van Mil, H.G.J., Richardson, M.K., 2011. Large-Scale assessment of the zebrafish embryo as a possible predictive model in toxicity testing. PLoS ONE 6. doi:10.1371/journal.pone.0021076

Ammar, S., Abdelhedi, R., Flox, C., Arias, C., Brillas, E., 2006. Electrochemical degradation of the dye indigo carmine at boron-doped diamond anode for wastewaters remediation. Environmental Chemistry Letters 4, 229-233. doi:10.1007/s10311-006-0053-2

Basheer, A.A., 2018. New generation nano-adsorbents for the removal of emerging contaminants in water. Journal of Molecular Liquids 261, 583-593. doi:10.1016/j.molliq.2018.04.021

Bide, M., 2007. Environmentally responsible dye application. Environmental Aspects of Textile Dyeing 74-92. doi:10.1533/9781845693091.74

Callieri, C., 2014. Picophytoplankton in Freshwater Ecosystems: The Importance of Small-Sized Phototrophs. Freshwater Reviews 1, 1-28. doi:10.1608/frj-1.1.1

CHARLES B. KIMMEL, WILLIAM W. BALLARD*, SETH R. KIMMEL, BONNIE ULLMANN, A.T.F.S., 1995. ZFIN Embyonic Developmental Stages. Developmental Dynamics 10, 253-310.

Chen, Z.X., Jin, X.Y., Chen, Z., Megharaj, M., Naidu, R., 2011. Removal of methyl orange from aqueous solution using bentonite-supported nanoscale zero-valent iron. Journal of Colloid and Interface Science 363, 601-607. doi:10.1016/j.jcis.2011.07.057

Deng, J., Dong, H., Zhang, C., Jiang, Z., Cheng, Y., Hou, K., Zhang, L., Fan, C., 2018. Nanoscale zero-valent iron/biochar composite as an activator for Fenton-like removal of sulfamethazine. Separation and Purification Technology 202, 130-137. doi:10.1016/j.seppur.2018.03.048 
Fan, J., Guo, Y., Wang, J., Fan, M., 2009. Rapid decolorization of azo dye methyl orange in aqueous solution by nanoscale zerovalent iron particles. Journal of Hazardous Materials 166, 904-910. doi:10.1016/j.jhazmat.2008.11.091

Fu, F., Dionysiou, D.D., Liu, H., 2014. The use of zero-valent iron for groundwater remediation and wastewater treatment: A review. Journal of Hazardous Materials 267, 194-205. doi:10.1016/j.jhazmat.2013.12.062

Garg, S., Jiang, C., Waite, T.D., 2018. Impact of pH on Iron Redox Transformations in Simulated Freshwaters Containing Natural Organic Matter. Environmental Science and Technology 52, 13184-13194. doi:10.1021/acs.est.8b03855

Guatame-Garcia, A. Buxton, M., 2018. The Use of Infrared Spectroscopy to Determine the Quality of Carbonate-Rich Diatomite Ores. Minerals 8, 120. doi: $10.3390 / \min 8030120$

Jung, K.Y., Lee, K.-L., Im, T.H., Lee, I.J., Kim, S., Han, K.-Y., Ahn, J.M., 2016. Evaluation of water quality for the Nakdong River watershed using multivariate analysis. Environmental Technology \& Innovation 5, 67-82. doi:10.1016/j.eti.2015.12.001

Khelifi, E., Gannoun, H., Touhami, Y., Bouallagui, H., Hamdi, M., 2008. Aerobic decolourization of the indigo dye-containing textile wastewater using continuous combined bioreactors. Journal of Hazardous Materials 152, 683-689. doi:10.1016/j.jhazmat.2007.07.059

Krysanov, E.Y., Pavlov, D.S., Demidova, T.B., Dgebuadze, Y.Y., 2010. Effect of Nanoparticles on Aquatic Organisms. doi:10.1134/S1062359010040114

Lowe, B.M., Skylaris, C.K., Green, N.G., 2015. Acid-base dissociation mechanisms and energetics at the silica-water interface: An activationless process. Journal of Colloid and Interface Science 451, 231-244. doi:10.1016/j.jcis.2015.01.094

Machado, G.E., Pereyra, A.M., Rosato, V.G., Moreno, M.S., Basaldella, E.I., 2019. Improving the biocidal activity of outdoor coating formulations by using zeolitesupported silver nanoparticles. Materials Science and Engineering C 98, 789-799. doi:10.1016/j.msec.2019.01.040

Nosrati, A., Larsson, M., Lindén, J.B., Zihao, Z., Addai-Mensah, J., Nydén, M., 2017. Polyethyleneimine functionalized mesoporous diatomite particles for selective copper recovery from aqueous media. International Journal of Mineral Processing 166, 29-36. doi:10.1016/j.minpro.2017.07.001

Pacheco-Álvarez, M.O.A., Picos, A., Pérez-Segura, T., Peralta-Hernández, J.M., 2019. Proposal for highly efficient electrochemical discoloration and degradation of azo dyes with parallel arrangement electrodes. Journal of Electroanalytical Chemistry 838, 195-203. doi:10.1016/j.jelechem.2019.03.004

Qiang, L., Cheng, J., 2019. Exposure to microplastics decreases swimming competence in larval zebrafish (Danio rerio). Ecotoxicology and Environmental Safety 176, 226-233. doi:10.1016/j.ecoenv.2019.03.088

Quinn, J., Geiger, C., Clausen, C., Brooks, K., Coon, C., O’Hara, S., Krug, T., Major, D., Yoon, W.S., Gavaskar, A., Holdsworth, T., 2005. Field demonstration of DNAPL dehalogenation using emulsified zero-valent iron. Environmental Science 
and Technology 39, 1309-1318. doi:10.1021/es0490018

Răducan, A., Puiu, M., Oancea, P., Colbea, C., Velea, A., Dinu, B., Mihăilescu, A.M., Galaon, T., 2019. Fast decolourization of Indigo Carmine and Crystal Violet in aqueous environments through micellar catalysis. Separation and Purification Technology 210, 698-709. doi:10.1016/j.seppur.2018.08.052

Raychoudhury, T., Naja, G., Ghoshal, S., Ortiz De La Plata, G.B., Alfano, O.M., Cassano, A.E., Cook, S.M., USEPA, Jia, H., Wang, C., Taylor, P., Greenlee, L.F., Hooker, S.A., Wang, L.-S., Wang, L.-S., Wang, L.-S., Wang, G., Li, Z.-H., Wang, J.-J., Goldstein, N., Greenlee, L.F., Yuvakkumar, R., Elango, V., Rajendran, V., Kannan, N., Singh, R.R.P.R.R.P.R.R.P., Misra, V., Singh, R.R.P.R.R.P.R.R.P., Liu, R., Lal, R., Ponder, S.M., Darab, J.G., Bucher, J., Caulder, D., Craig, I., Davis, L., Edelstein, N., Lukens, W., Nitsche, H., Rao, L., Shuh, D.K., Mallouk, T.E., Cirtiu, C.M., Raychoudhury, T., Ghoshal, S., Moores, A., Moore, K., Forsberg, B., Baer, D.R., Arnold, W. a., Penn, R.L., Li, X., Elliott, D.W., Zhang, W., Oropeza, S., Corea, M., Gómez-Yáñez, C., Cruz-Rivera, J.J., NavarroClemente, M.E., Epa, 2012. Preparation and characterization of zero valent Iron nanoparticles. Available online (last verified July 27, 2012): http://www.epa.gov/rpdweb00/docs/cleanup/nanotechnology/chapter-2-zerovalent.pdf 137, 53-59. doi:10.1007/s10661-011-2213-5

Saleh, S.M., 2019. ZnO nanospheres based simple hydrothermal route for photocatalytic degradation of azo dye. Spectrochimica Acta - Part A: Molecular and Biomolecular Spectroscopy 211, 141-147. doi:10.1016/j.saa.2018.11.065

Samchetshabam, G., Hussan, A., Choudhury, T.G., 2017. Impact of Textile Dyes Waste on Aquatic Environments and its Treatment Impact of Textile Dyes Waste on Aquatic Environments and its Treatment.

Shah, S.Z.H., Khan, U., Riaz, S., Naseem, S., 2015. Effect of pH on Iron Oxide Nanoparticles, Materials Today: Proceedings. Elsevier Ltd. doi:10.1016/j.matpr.2015.11.024

Sharma, H., Shirkot, P., 2019. Bioremediation of azo dyes using biogenic iron nanoparticles 7, 12-15. doi:10.15406/jmen.2019.07.00232

Wang, F., Huang, J., Xu, J., 2018. Continuous-flow synthesis of azo dyes in a microreactor system. Chemical Engineering and Processing - Process Intensification 127, 43-49. doi:10.1016/j.cep.2018.03.014

Westerfield, M., 2000. A guide for the laboratory use of zebrafish (Danio rerio), 4th ed., U. ed. BMC Dev Biol.

Wu, G., Cheng, Y., Ren, Y., Wang, Y., Wang, Z., Wu, H., 2015. Synthesis and characterization of $\gamma$-Fe2O3@C nanorod-carbon sphere composite and its application as microwave absorbing material. Journal of Alloys and Compounds 652, 346-350. doi:10.1016/j.jallcom.2015.08.236

Yuan, P., Liu, D., Fan, M., Yang, D., Zhu, R., Ge, F., Zhu, J.X., He, H., 2010. Removal of hexavalent chromium [Cr(VI)] from aqueous solutions by the diatomitesupported/unsupported magnetite nanoparticles. Journal of Hazardous Materials 173, 614-621. doi:10.1016/j.jhazmat.2009.08.129

Zhang, M., Bacik, D.B., Roberts, C.B., Zhao, D., 2013. Catalytic hydrodechlorination 
